\title{
Under nutrition and associated factors among school adolescents in Dangila Town, Northwest Ethiopia: a cross sectional study
}

\author{
Yeshalem Mulugeta Demilew, Amanu Aragaw Emiru
}

School of Public Health, College of Medicine and Health Sciences, Bahir Dar University P.O.Box 79, Bahir Dar, Ethiopia.

E-mail: Amanu Aragaw Emiru: amanuaragaw@yahoo.com

\begin{abstract}
Background: Under nutrition in adolescents is an important determinant of health outcomes. Yet, adolescents are not usually part of health and nutrition surveys. Therefore, this research was conducted to assess factors associated with under nutrition among school adolescents.

Method: A cross-sectional study was conducted among 424 school adolescents from November 1-15, 2015. Simple random sampling was used to select the study participants. Data were collected using structured questionnaire and analyzed by SPSS version 20 software. Both bivariate and multivariable logistic regression analyses were carried out to identify predictors of under nutrition. Furthermore, anthropometric data were calculated using Anthro-plus software.

Result: The prevalence of stunting and thinness were $24.8 \%$ and $7.1 \%$, respectively. Male gender [AOR=3.2, $95 \%$ CI: $(1.7$, $5.8)]$, infrequent food intake [AOR=4.6, 95\% CI: $(2.6,8.0)]$, unavailability of latrine [AOR=2.7, $95 \% \mathrm{CI}:(1.2,6.0)]$, and poor hand washing practice $[\mathrm{AOR}=3.9,95 \% \mathrm{CI}:(1.9,8.1)]$ were independent predictors of stunting. Factors associated with thinness were being male $[\mathrm{AOR}=11.5,95 \% \mathrm{CI}:(3.3,39.5)]$, illness in the last two weeks $[\mathrm{AOR}=2.9,95 \% \mathrm{CI}:(1.2,7.0)]$, and having more than five family members [AOR $=3.6,95 \% \mathrm{CI}:(1.3,9.4)]$.

Conclusion: The prevalence of under nutrition was high in this study. Infrequent food intake, unavailability of a latrine, poor hand washing practice, and large family members were the factors associated with under nutrition. There is need to implement nutrition education to school adolescents by giving emphasis on environmental and personal hygiene.
\end{abstract}

Keywords: Adolescent, stunting, thinness.

DOI: https://dx.doi.org/10.4314/ahs.v18i3.34

Cite as: Demilew YM, Emiru AA. Under nutrition and associated factors among school adolescents in Dangila Town, Northwest Ethiopia: a cross sectional study. Afri Health Sci. 2018;18(3): 756-766. https:/ / dx.doi.org/10.4314/abs.v18i3.34

\section{Background}

The World health organization (WHO) defined adoles- cents as individuals between the ages of 10-19 years ${ }^{1}$. Adolescence, a period of transition from childhood to adulthood, is crucial in the life of human beings and characterized by an exceptionally rapid rate of growth ${ }^{2,3}$. This period is known to be a second opportunity for growth, as it facilitates catch up growth for children experiencing nutritional deficits in their early life $\mathrm{f}^{4,5}$.

Due to rapid growth, during this period, the dietary requirement of adolescents is higher for most nutrients than at any other period of life $^{2}$. If their requirement

E-mail: yeshalem_mulugeta@yahoo.com

(C) 2018 Demilew et al. Licensee African Health Sciences. This is an Open Access article distributed under the terms of the Creative commons Attribution License (https://creativecommons.org/licenses/BY/4.0), which permits unrestricted use, distribution, and reproduction in any medium, provided the original work is properly cited. 
is not met, at this age, they are likely to develop serious nutritional deficiency ${ }^{2}$. Despite the economic growth observed in developing countries, under nutrition is still highly prevalent among school-going adolescents ${ }^{6-10}$.

Under nutrition during adolescence is an important determinant of health outcomes. Apart from causing significant mortality, it creates long-lasting effect on growth, development and physical fitness of survivors ${ }^{10-13}$. This, in turn, affects their ability to learn and work at maximal productivity $^{14,15}$. In girls, short stature may persist into adulthood and increase the risk of adverse reproductive outcomes ${ }^{16}$.

Therefore, nutritional support during this period of life affects not only the individual wellbeing of adolescents but also the well-being of entire societies ${ }^{17}$. Ensuring that adolescents can successfully navigate this phase of life will help to break the cycle of poverty and produce benefits for individuals, communities, and nations ${ }^{10,18}$. However, Ethiopian adolescents received low priority in terms of nutrition intervention ${ }^{2}$.

To address nutritional problems of adolescents, it is crucial to assess their current nutritional status. Thus, the present study was undertaken to ascertain the level of under nutrition and associated factors among school adolescents. Findings obtained from this study may assist decision makers to plan and implement nutrition intervention programs to benefit school adolescents.

\section{Methods \\ Study setting}

The study was conducted in Dangila town, Northwest Ethiopia. The town is located $484 \mathrm{~km}$ Northwest of Addis Ababa (capital city of Ethiopia). In Ethiopia, education curriculum is classified into four cycles such as the primary school with first (grade 1-4) and second (grade 5-8) cycles; and secondary school with first (grade 9-10) and second (grade 11-12) cycles. This study was conducted among secondary school first cycle (grade 9-10) students. In the town, there was one first cycle secondary school with a total of 2,867 students during the time of the study, of which 1,504 were females.

\section{Study design and population}

A school based cross-sectional study was conducted from November 1 to 15, 2015. Adolescents above fifteen years old were included in the study. However, those who were transferred from other schools during the academic year of data collection period were excluded from our study.

\section{Sample size, sampling procedure, and data collec- tion}

The sample size was determined using single population proportion formula, with the following assumptions: a 95\% confidence level, 5\% marginal error, $50 \%$ proportion of under nutrition as there was no previous study, $10 \%$ non-response rate. The sample size was 424 .

The sampling frame (list of students) was obtained from school registration book. Using this registration log book, study participants were selected by simple random sampling technique (lottery method).

Data were collected using a self-administered questionnaire adapted by reviewing literature ${ }^{20}$. The questionnaire was first developed in English then translated into Amharic (local language), and then back to English to check consistency. The questionnaire focused on socio-demographic characteristics (sex, age, residence, grade, father and mother education, father and mother occupation, living arrangement and the number of family members), availability of latrine, source of drinking water, frequency of food intake, type of food they took frequently, and hand washing practice.

Four diploma nurses and one public health professional were recruited as data collectors and supervisor, respectively. The data collectors and supervisor were trained for two days on the purpose of the study, data collection technique, and tools. The study participants were not allowed to discuss their responses with each other to avoid shared responses.

Anthropometric measurements were done at the school premise. Each participant was measured for weight and height. Weight was measured to the nearest $0.1 \mathrm{~kg}$ using a digital weight scale. During weight measurement, each participant wore light clothes. Likewise, height was measured to the nearest $0.1 \mathrm{~cm}$ using a stadiometer. During the measurement, each participant stood on the measuring board without shoes, considering the normal anatomical position, and heels, buttocks, shoulders, and back of the head touching the board. The flat headpiece of the measuring board touched the crown of the head and formed a right angle. For both height and weight two 
readings were recorded, and the computed average was used in the analysis. Body mass index was calculated as the ratio of weight in kilograms to the square of height in meters.

The z-score values for BMI-for-age and height for age were calculated using WHO Anthro-Plus software (21). Calculated z-scores of BMI-for-age and height for age were used to classify thinness and stunting using the new WHO 2007 reference value. The z-score values for BMIfor-age were interpreted as follows: adolescents who scored $<-3 \mathrm{SD}$, between $-2 \mathrm{SD}$ to $-3 \mathrm{SD},-2 \mathrm{SD}$ to $+2 \mathrm{SD}$, and $>+2$ SD were declared severely thin, thin, normal, and overweight, respectively. Similarly, the z-score values for height-for-age were interpreted as follows: adolescents who scored below $-3 \mathrm{SD}$, between -2SD to -3SD, $>-2 \mathrm{SD}$ stated as severely stunted, stunted, and normal, respectively. Those adolescents who had stunting and/or thinness were considered as undernourished.

\section{Data quality control}

Data collectors and the supervisor were trained on the purpose of the study, data collection technique and tool before and after pretesting. The questionnaire was pre-tested among 43 students in a school where the study was not undertaken, and modifications were done accordingly. The weighing scale and height measuring stadiometer were checked after each measurement. Each day, the collected data were reviewed and errors were returned back to the data collectors for correction. Data validity and reliability was maintained through close supervision of data collection process by the supervisor and investigators.

\section{Data processing and analysis}

Data were double entered and analyzed using SPSS ver- sion 20 software. The frequency of each variable was calculated to check for accuracy, outliers, consistency and missed values. The proportion of under nutrition was determined. Crude and adjusted Odds ratios were computed for each explanatory variable to determine the strength of association and control confounders. The p-value $<0.2$ was taken as a cut-off point to select variables for the multivariable logistic regression models. The final model was built using backward elimination. The p-values less than 0.05 was considered statistically significant.

\section{Ethical consideration}

The study was approved by the Ethical Review Committee of Amhara Regional Health Bureau. Letter of permission was taken from the school administrator. Consent was obtained from school directors, and assent was taken from every participant. Privacy and confidentiality were maintained throughout the study period by excluding personal identifiers from the data collection form.

\section{Result}

\section{Socio-demographic characteristics of the adoles- cents and their parents}

Of 424 adolescents participated in the study, complete data were obtained among 407 participants, making the response rate $95.9 \%$. The mean (+SD) age of the respondents was $16.67 \pm(0.93)$ years. The majority, 394 $(96.8 \%)$ of the participants were Orthodox Christian followers, and $350(86 \%)$ were Amhara in ethnicity (Table1). Two hundred seventy $(66.3 \%)$ of the respondents' fathers, and 287(70.5\%) of their mothers, had no formal education. Whereas, only $44(10.8 \%)$ of their fathers and $34(8.4 \%)$ of their mothers attended beyond secondary education. About six in ten of their fathers, 251(61.7\%) and their mothers, 235(57.7) were farmers and housewives in their profession (Table 1). 
Table1: Socio- demographic characteristics of school adolescents and their parents in Dangila town, 2015.

\begin{tabular}{|c|c|c|}
\hline Variable & Frequency $(n=407)$ & Percentage $(\%)$ \\
\hline \multicolumn{3}{|l|}{ Religion } \\
\hline Orthodox & 394 & 96.8 \\
\hline Muslim & 7 & 1.7 \\
\hline Protestant & 6 & 1.5 \\
\hline \multicolumn{3}{|l|}{ Sex } \\
\hline Male & 188 & 46.2 \\
\hline Female & 219 & 53.8 \\
\hline \multicolumn{3}{|l|}{ Ethnicity } \\
\hline Amhara & 350 & 86 \\
\hline Tigrie & 47 & 11.5 \\
\hline Agew & 10 & 2.5 \\
\hline \multicolumn{3}{|c|}{ Respondents currently live with } \\
\hline Both parents & 266 & 65.4 \\
\hline Mother & 50 & 12.3 \\
\hline Alone & 26 & 6.4 \\
\hline Relatives & 23 & 5.7 \\
\hline Friends & 36 & 8.8 \\
\hline Father & 6 & 1.5 \\
\hline \multicolumn{3}{|l|}{ places of residence } \\
\hline Urban & 185 & 45.5 \\
\hline Rural & 222 & 54.5 \\
\hline \multicolumn{3}{|c|}{ Educational status of the father } \\
\hline Have no formal education & 270 & 66.3 \\
\hline Primary education & 42 & 10.3 \\
\hline Secondary education & 51 & 12.5 \\
\hline Above secondary education & 44 & 10.8 \\
\hline \multicolumn{3}{|c|}{ Occupational status of the father } \\
\hline Daily laborer & 37 & 9.1 \\
\hline Farmer & 251 & 61.7 \\
\hline Government employee & 67 & 16.5 \\
\hline Merchant & 41 & 10.1 \\
\hline Driver & 11 & 2.7 \\
\hline \multicolumn{3}{|c|}{ Educational status of the mother } \\
\hline Have no formal education & 287 & 70.5 \\
\hline Primary education & 39 & 9.6 \\
\hline Secondary education & 47 & 11.5 \\
\hline Above secondary education & 34 & 8.4 \\
\hline \multicolumn{3}{|c|}{ Occupational status of the mother } \\
\hline Housewife & 235 & 57.7 \\
\hline Daily laborer & 40 & 9.8 \\
\hline Farmer & 74 & 18.2 \\
\hline Government employee & 33 & 8.1 \\
\hline Merchant & 25 & 6.1 \\
\hline
\end{tabular}


Eating behavior and hygienic practices of the respondents

More than half, 207(56.3\%) of the adolescents consumed "maize and millet mix injera" with "Wot" as a daily staple

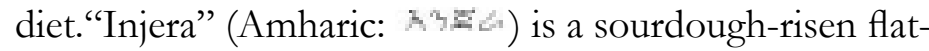
bread with a slightly spongy texture. "Wot”(Amharic: 㲅) is an Ethiopian stew or curry that may be prepared with chicken, beef, lamb, a variety of vegetables, legumes, and spice mixtures such as berebere (matured green pepper). Two thirds, $270(66.3 \%)$ of the respondents often took three or more meals per day. The majority of the adolescents used piped water for drinking, 351(86.2\%) and had a functional latrine at their home, 336(82.6\%). More than three fourths, $318(78.1 \%$ ) of the adolescents had the habit of washing their hand before eating (Table 2).

\section{Table 2: Eating behavior and sanitation practices of adolescents, Dangila Town, Northwest Ethiopia, 2015}

\begin{tabular}{lll}
\hline Variable & Frequency & Percentage \\
\hline Kind of food always eaten at home & & \\
Maize \& millet injera with "wot" & 207 & 50.9 \\
Teffinjera with "wot" & 178 & 43.7 \\
Wheat, maize, millet and Teffinjera with "wot" & 22 & 5.4 \\
Number of feeding per day & & \\
1-2 times & 137 & 66.3 \\
3-4 times & 270 & 33.7 \\
Use iodized salt & & \\
Yes & 250 & 61.4 \\
No & 157 & 38.6 \\
Wash hands with soap before eating & & \\
Yes & 318 & 78.1 \\
No & 89 & 21.9 \\
Source of drinking water & 351 & 86.2 \\
Pipe \& protected spring & 56 & 13.8 \\
River & & \\
Availability of functional latrine at home & 336 & 82.6 \\
Yes & 71 & 17.4 \\
No
\end{tabular}

Nutritional status of school adolescents

About 101(24.8\%), and 33 (8.1\%) school adolescents were stunted and severely stunted, respectively. The prev- alence of thinness was $7.1 \%$. More boys than girls were stunted (36.1\% versus $15.0 \%$ ) and thin (13.8 versus $1.4 \%)$ in this study (Table 3). 
Table 3: prevalence of under nutrition among school adolescents in Dangila town from November 01-05/2015.

\begin{tabular}{lllll}
\hline Variable & Male & Female & Total & $\mathbf{X}^{2}($ P-value) \\
\hline Stunted & & & & \\
Yes & $68(36.2)$ & $33(15.1)$ & $101(24.8)$ & $24.143(<0.001)$ \\
No & $120(63.8)$ & $186(45.7)$ & $306(75.2)$ & \\
Severely stunted & & & & \\
Yes & $22(11.7)$ & $11(5.0)$ & $33(8.1)$ & $6.057(0.014)$ \\
No & $166(88.3)$ & $208(95.0)$ & $374(91.9)$ & \\
Thinness & & & & \\
Yes & $26(13.8)$ & $3(1.4)$ & $29(7.1)$ & $23.732(<0.001)$ \\
No & $162(86.2)$ & $216(98.6)$ & $378(92.9)$ & \\
\hline
\end{tabular}

Factors associated with under nutrition of adolescents

The bivariate logistic regression analysis showed that being a male, lack of possession of television and radio, being from a rural residence, taking less than three meals per day, unavailability of latrine and lack of hand washing practice were statistically associated with stunting (Table 4). Factors associated with thinness were being a male, having illness in the last two weeks, drinking river water, and living in the household having more than five family members (Table 5).

Table 4: Factors associated with stunting among school adolescents in Dangila town, 2015.

\begin{tabular}{|c|c|c|c|c|}
\hline \multirow[t]{2}{*}{ Factor } & \multicolumn{2}{|c|}{ Stunting } & \multirow{2}{*}{$\begin{array}{l}\text { COR } \\
(95 \% \mathrm{C} / \mathrm{I})\end{array}$} & \multirow{2}{*}{$\begin{array}{l}\text { AOR } \\
(95 \% \mathrm{C} / \mathrm{I})\end{array}$} \\
\hline & Yes & No & & \\
\hline \multicolumn{5}{|l|}{ Sex } \\
\hline Male & $68(16.7)$ & $120(29.5)$ & $3.1(1.9,5.1)$ & $3.2(1.7,5.8)$ \\
\hline Female & $33(8.1)$ & $186(45.7)$ & 1.00 & 1.00 \\
\hline \multicolumn{5}{|l|}{ Area of parental residence } \\
\hline Urban & $36(8.8)$ & $149(36.6)$ & 1.00 & \\
\hline Rural & $65(16.0)$ & $157(38.6)$ & $1.7(1.0,2.7)$ & \\
\hline \multicolumn{5}{|l|}{ Possession of television } \\
\hline Yes & $20(4.9)$ & $104(25.6)$ & 1.00 & \\
\hline No & $81(19.9)$ & $202(49.6)$ & $2.0(1.2,3.5)$ & \\
\hline \multicolumn{5}{|l|}{ Possession of radio } \\
\hline Yes & $49(12.0)$ & $207(50.9)$ & 1.00 & $2.0(1.1 ., 3.5)$ \\
\hline No & $52(12.8)$ & $99(24.3)$ & $2.2(1.4,3.5)$ & 1.00 \\
\hline \multicolumn{5}{|l|}{ Frequency of food intake per day } \\
\hline $1-2$ times & $64(15.7)$ & $73(17.9)$ & $5.5(3.4,8.9)$ & $4.6(2.6,8.0)$ \\
\hline $3-4$ times & $37(9.1)$ & $233(57.3)$ & 1.00 & 1.00 \\
\hline \multicolumn{5}{|l|}{ Wash their hand by soap and water } \\
\hline Yes & $51(12.5)$ & $38(9.3)$ & $7.1(4.2,12.0)$ & $3.9(1.9,8.1)$ \\
\hline \multicolumn{5}{|l|}{ No } \\
\hline $\begin{array}{l}\text { Availability of functional latrine at } \\
\text { home }\end{array}$ & $59(14.5)$ & $277(68.1)$ & 1.00 & 1.00 \\
\hline Yes & $42(10.3)$ & $29(7.1)$ & $6.8(3.9,11.7)$ & $2.7(1.2,6.0)$ \\
\hline No & & & & \\
\hline
\end{tabular}


Table 5: Factors associated with thinness among school adolescents in Dangila town, 2015.

\begin{tabular}{lllll}
\hline Factor & \multicolumn{2}{c}{ Underweight } & $\begin{array}{l}\text { COR } \\
\mathbf{( 9 5 \% C / I )}\end{array}$ & $\begin{array}{l}\text { AOR } \\
\mathbf{( 9 5 \%} \mathbf{C} / \mathbf{I})\end{array}$ \\
\cline { 2 - 3 } & Yes & No & $11.5(3.4,38.8)$ & $11.5(3.3,39.5)$ \\
\hline $\begin{array}{l}\text { Sex } \\
\text { Male }\end{array}$ & $26(6.4)$ & $162(39.8)$ & 1.00 & 1.00 \\
$\begin{array}{l}\text { Female } \\
\text { Source of drinking water }\end{array}$ & $3(0.7)$ & $216(53.1)$ & & \\
$\begin{array}{l}\text { Pipe and protected spring } \\
\text { River }\end{array}$ & $21(5.2)$ & $330(81.1)$ & 1.00 & \\
$\begin{array}{l}\text { Illness in the last two weeks } \\
\text { Yes }\end{array}$ & $8(2.0)$ & $48(11.8)$ & $2.6(1.0,6.2)$ & \\
No & $12(2.9)$ & $69(17.0)$ & 1.00 & 1.00 \\
Family members & $17(4.2)$ & $309(75.9)$ & $3.1(1.4,6.9)$ & $2.9(1.2,7.0)$ \\
$\leq 5$ & & & & \\
$>5$ & $6(1.5)$ & $202(49.6)$ & 1.00 & 1.00 \\
\hline
\end{tabular}

COR- Crude odds ration; AOR - adjusted odds ratio, C/I- Confidence interval

In the multivariable logistic regression analysis, a significant association was observed between stunting and sex, in which males were 3.1 times more likely to be stunted than girls $[\mathrm{AOR}=3.2,95 \% \mathrm{CI}:(1.7,5.8)]$. The frequency of food intake had association with stunting, adolescents who took food two or less times per day were 4.6 times more likely to be stunted than those who took more than two times per day $[\mathrm{AOR}=4.6,95 \% \mathrm{CI}:(2.6,8.0)]$.

Hand washing practice was another predictor for stunting, adolescents who did not wash their hands before eating were almost 4 times more likely to be stunted compared to those adolescents who did wash their hands $[\mathrm{AOR}=3.9,95 \% \mathrm{CI}=1.9,8.1]$. Unavailability of latrine had a significant association with stunting, adolescents from families who did not have latrine were 2.7 times more likely to be stunted compared with families who had latrine $[\mathrm{AOR}=2.7,95 \% \mathrm{CI}:(1.2,6.0)]$. The odds of having stunting was 2 times higher among adolescents whose family had no radio as compared to their counterparts $[\mathrm{AOR}=2.0,95 \% \mathrm{CI}:(1.1,3.5)]$ (Table 4).

The number of family members was one of the strongest predictors for thinness, adolescents who lived in the household with more than 5 family members were 3.6 times more likely to be thinner than those who lived in the household with five or less family size $[\mathrm{AOR}=3.6$, $95 \%$ CI: $(1.3,9.4)]$. Being a male gender had a significant association with thinness, male adolescents were 11.5 times more likely to be thinner compared with female adolescents $[\mathrm{AOR}=11.5,95 \% \mathrm{CI}:(3.3,39.5)]$. Having ill- ness had also a significant association with thinness, adolescents who had illness within fifteen days prior to the study were almost three folds more likely to be thinner than their counterparts $[\mathrm{AOR}=2.9,95 \% \mathrm{CI}:(1.2,7.0)]$ (Table 5).

\section{Discussion}

The aim of this study was to assess nutritional status and associated factors among school adolescents. In view of that, in this study, the prevalence of stunting was high $(24.8 \%)$. More males than females were stunted ( $\mathrm{p}$-value, <0.001). This finding was almost comparable with the prevalence documented in Northern Ethiopia (28.5\%), Southwest Nigeria (19.1\%), and Northeastern Brazil $(20.7 \%)^{23-25}$.

The prevalence of stunting in this study was higher than a study finding in South-Western Nigeria $(15.7 \%)^{26}$. This discrepancy might be due to the difference in habit of food intake, socioeconomic status and cultural variation between the study subjects. On the other hand, the prevalence of stunting in our study was lower than the study findings in West Bengal, India (44-46.6\%) ${ }^{27,28}$. The difference might be due to time gap; currently, there is an implementation of nutrition intervention programs by governmental and nongovernmental organizations especially in the first two years of life. This intervention could have a direct contribution to improving the nutritional status of adolescents because stunting is due to the effect of malnutrition starting from the first 1000 days. 
In this study, $7.1 \%$ of the adolescents were thin. This prevalence was consistent with the study finding in Seychelles $(6.7 \%)^{29}$. However, the magnitude was lower compared to the study findings in Southeast Ethiopia (13.6\%), Northern Ethiopia (26.1\%), Nigeria (18.9\%) and West Bengal, India $(28 \%)^{25,26,28,30}$. The discrepancy might be due to the time gap between studies and current implementation of nutritional programs.

The likelihood of both stunting and thinness was higher among boys compared to girls. This result was in line with the study findings in different parts of Ethiopia and Nigeria, ${ }^{9,25,26,31-34}$. The reason for high prevalence of under nutrition among males than females might be related to biological, behavioral, and socio-cultural mechanisms.

Many studies throughout the world revealed the relationship between dietary intake and nutritional status of an individual ${ }^{30,35-38}$. Similarly, in this study, adolescents who took food two or less times per day were more likely to be stunted than those who took food more than two times per day. This might be explained due to the fact that infrequent taking of food is less likely to meet the nutritional requirement.

Adolescents who did not wash their hands before eating were more likely to be stunted compared to those adolescents who did wash their hands. This finding was in line with the study findings in different parts of Ethiopia and India ${ }^{38-40}$. Hand washing practice is essential to prevent diarrhea and other feco-oral diseases, which in turn may contribute to the reduction of under nutrition.

A significant association was observed between thinness and number of family members. This finding was comparable with the study finding in Northwest Ethiopia ${ }^{41}$. This might be due to the fact that large family size hampers food security status of the household ${ }^{42}$, which is an important determinant of nutritional status of the family ${ }^{43}$. The risk of being stunted was higher among adolescents from families who did not have latrine compared with families who had a latrine. A similar result was reported from previous study finding in Northwest Ethiopia ${ }^{41}$. This might be due to the fact that poor sanitation and hygiene interventions lead to repeated bouts of diarrhea and intestinal worms, which in turn deteriorates the nu- tritional status of adolescents ${ }^{44,45}$. The other explanation could be attributed to repeated fecal contamination that leads to environmental enteropathy, which is an inflammatory response, increases the small intestine's permeability to pathogens while reducing nutrient absorption ${ }^{46,47}$.

Adolescents who had illness within fifteen days prior to the study were more likely to be thin than their counterparts. Infection plays a major role in the etiology of under nutrition because of infection results in increased needs and high energy expenditure, lower appetite, utilization of nutrients and disruption of metabolic equilibrium and nutrient losses due to vomiting, diarrhea, poor digestion and mal-absorption ${ }^{48}$.

The odds of having stunting was higher in adolescents whose family had no a radio as compared to adolescents' families who possessed a radio. The result was in agreement with other studies ${ }^{41,49}$. Families who have radio are more likely to get nutrition-related message.

Random selection of participants, using well-trained sexmatched data collectors and validated data collection instruments were strengths of this study. Whereas, the cross-sectional design makes any inference of growth pattern over time difficult was the limitation of this study.

\section{Conclusion}

The prevalence of under nutrition was high in this study. Factors associated with stunting were being a male, lack of possession of a radio, unavailability of latrine, infrequent food intake and poor hand washing practice. Illness in the last two weeks, having more than five family members and being a male were positively associated with thinness. There is need to implement nutrition education to school adolescents by giving emphasis for environmental and personal hygiene.

\section{List of abbreviations}

BMI- Body Mass Index

WHO -World Health Organization

\section{Competing interests}

The authors declare that they have no competing interests 


\section{Funding}

This research was not funded by any organization

\section{Authors' contributions}

YM: Conceived and designed the study, conducted statistical analysis and result interpretation, prepared manuscript. The author read and approved the manuscript.

AA: assisted the study design, data analysis and result interpretation, prepared manuscript. The authors read and approved the manuscript.

\section{Acknowledgements}

The authors are indebted to study participants who participated in this study and provided valuable information with their full cooperation. We are also grateful to express our gratitude to data collection facilitators and the supervisor for their time and full commitment.

\section{Authors' information}

YM: BSC, MPH; I am working in Bahir Dar University, College of Medicine and Health Sciences, Bahir Dar, Ethiopia.

AA: BSC, MSC in Reproductive Health; I am working in Bahir Dar University, College of Medicine and Health Sciences, Bahir Dar, Ethiopia.

\section{Reference}

1. World Health Organization/UNICEF. A picture of Health: a review of annotated bibliography of the health of young people in developing countries. Geneva: WHO, 1995.

2. World Health Organization. Nutrition in adolescence - issues and challenges for the health sector. Geneva: WHO Press; 2005. ISBN 9241593660.

3. Anand K, Kant S, Kapoor SK. Nutritional status adolescents school children in rural North India. Indian Pediatrics. 1999;36(8):810-5.

4. Rao S. Nutritional Status of the Indian population. $J$ Biosci. 2001;26:481-9.

5. Golden M. Is complete catch-up possible for stunted malnourished children? Eur J Clin Nutr. 1994;48:S58-71.

6. Kurian J, Mahesh SH, Aravind AP, Kurian J. A study to determine the nutritional status of high school children in rural field practice area of medical college. International Journal of Contemporary Pediatrics. 2014;1(3):148-51.
7. Essien E, Emebu P, Iseh K, Haruna M. Assessment of Nutritional Status and Knowledge of Students from Selected Secondary Schools in Sokoto Metropolis, Sokoto State, Nigeria. African Journal of food, Agriculture, Nutrition and Development. 2014;14(6):9454-68.

8. Taru M, Hesham E-S, David TD, Jason RR. The prevalence of underweight, overweight, obesity and associated risk factors among school-going adolescents in seven African countries. Public Health. 2014. 2014;14(887): http:/ / www.biomedcentral.com/1471-2458/14/887.

9. Hadush G, Omer S, Huruy A. Assessment of Nutritional Status and Associated Factors among School Going Adolescents of Mekelle City, Northern Ethiopia. International Journal of Nutrition and Food Sciences. 2015;4(1):11824: doi: 10.11648/j.ijnfs.20150401.26.

10. UNICEF. Progress for Children A report card on adolescents. United Nations Plaza, New York, NY 10017, USA: 2012.

11. Howard EK, Nimrod B, Dominic M, Steven S. The effect of chronic childhood malnutrition on pubertal growth and development. Am J Clin Nutr. 1982;36:52736.

12. Blössner, Monika, de Onis, Mercedes. Malnutrition: quantifying the health impact at national and local levels. Geneva, World Health Organization, 2005. (WHO Environmental Burden of Disease Series, No. 12).

13. John S, SmithTrevor. Malnutrition: causes and consequences. Clinical Medicine. 2010;10(6):624-7.

14. Vinicius JBM, Telma MMTF, Luciane PG, Maria dCPF, Paula AM, Paula GCA, et al. Long-Lasting Effects of Undernutrition. Int J Environ Res Public Health. 2011;8: 1817-46; doi:10.3390/ijerph8061817.

15. Essien E, Haruna MJ, Emebu PK. Prevalence of Malnutrition and its Effects on the Academic Performance of Students in Some Selected Secondary Schools in Sokoto Metropolis. Pakistan Journal of Nutrition. 2012;11(7):511-5, ISSN 1680-5194.

16. Afework M, Fitsum H, Barbara S, Gideon K, Vincent L, Zenebe A, et al. Nutritional Status of Adolescent Girls from Rural Communities of Tigray, Northern Ethiopia. Ethiopian Journal of Health Development. 2009;23(1):5.

17. Dean TJ, Joel GB, Anthony RM, George A, Mariam C, David BE, et al., editors. Disease Control Priorities in Developing Countries 2nd edition. Chapter 58Schoolbased Health and Nutrition Programs. Washington (DC: World Bank; 2006. 
18. UnitedNationsPopulationFund(UNFPA). Status Report.Adolescents and Young People in Sub-Saharan Africa:Opportunities and Challenges. Washington, DC:USA: 2012.

19. Doustmohammadian A, Dorostymotlagh AR, Keshavarz A, Sadrzadehyeganeh H, Mohammadpour-Ahrangani B. Socio-demographic factors associated with body mass index of female adolescent students in Semnan city, Iran. Malaysian Journal of Nutrition. 2009;15(1):27-35.

20. Dawit D, Abraham D, Abebe A. Undernutrition and associated risk factors among school age children in Addis Ababa, Ethiopia. BMC Public Health. 2015;15(375).

21. CentralStatisticalAgency[Ethiopia], ICFInternational. Ethiopia Demographic and Health Survey 2011. Addis Ababa, Ethiopia and Calverton, Maryland, USA: Central Statistical Agency and ICF International. 2012.

22. Muller O, Krawinkel M. Malnutrition and health in developing countries. CMAJ. 2005;173(3):279-86.

23. Idowu OS, Kazeem AO, Olumuyiwa OO, Olisamedua FN. Prevalence of and Risk factors for Stunting among School Children and Adolescents in Abeokuta, Southwest Nigeria. Journal of Health and Population Nutrition. 2011;29(4):364-70.

24. Elyssia Karine Nunes MR, Risia Cristina EdM, Juliana SO, Alice AOM, Tatiane LT, Giovana L-S, et al. Nutritional status of children and adolescents from a town in the semiarid Northeastern Brazil. Revista Paulista Pediatria. 2014;32(3):200-7.

25. Yohannes AM, Gordon AZ, Tiffany KG, Robert JA, Zumin S. Prevalence and factors associated with stunting and thinness among adolescent students in Northern Ethiopia: a comparison to World Health Organization standards. Archives of Public Health. 2015;73(44): DOI 10.1186/s13690-015-0093-9.

26. Olayinka OO, Kofoworola IA, Joshua OA, Akinyinka OO, Babatunde OA, Oluyomi E. Gender and Rural-Urban Differences in the Nutritional Status of In-School Adolescents in South-Western Nigeria. Journal of Biosocial Science. 2010;42:653-76.

27. Nitish M, Jaydip S. Prevalence of stunting and thinness among rural adolescents of Darjeeling district, West Bengal, India. JPH. 2010;7(1):55-61.

28. Dey I, Biswas R, Ray K, Bhattacherjee S, Chakraborty M, Pal P. Nutritional status of school going adolescents in a rural block of Darjeeling, West Bengal, India. The Health. 2011;2(3):75-7.
29. Abudayya A, Shi Z, Abed Y, Holmboe-Ottesen G. Diet, nutritional status and school performance among adolescents in Gaza Strip. Eastern Mediterranean Health Journal. 2011;17(3):218-25.

30. Ahmed YM, Tomas BT. Nutritional Status and Associated Risk Factors Among Adolescent Girls in Agarfa High School, Bale Zone, Oromia Region, South East Ethiopia. International Journal of Nutrition and Food Sciences. 2015;4(4):445-52.

31. Huruy A, Tefera B, Legesse N. Socioeconomic Factors Associated with Underweight and Stunting among Adolescents of Jimma Zone, South West Ethiopia: A Cross-Sectional Study. Hindawi Publishing Corporation. 2013; Article ID 238546, 7 pages,http://dx.doi. org/10.1155/2013/238546.

32. Tolessa DD, Mektie W, Aderajew NT. Nutritional status and associated factors among school adolescent in Chiro Town, West Hararge, Ethiopia. Gaziantep Medical Journal. 2015;21(1):32-42.

33. Olumakaiye MF. Prevalence of Underweight:A Matter of Concern among Adolescents in Osun State, Nigeria. Pakistan Journal of Nutrition. 2008;7(3):503-8.

34. Olayinka OO, Kofoworola IA, Joshua OA, Akinyinka OO, Babatunde OA, Oluyomi E. Gender and rural- urban differences in the nutritional status of in-school adolescents in south-western Nigeria. J Biosoc Sci. 2010;42:65376: doi:10.1017/S0021932010000234.

35. Kodavalla V, Narsimhachary VBG, Kamasamudram V. Identification of dietary patterns by factor analysis and study of the relationship with nutritional status of rural adolescents using factor scores. Journal of Health, Population and Nutrition. 2015;34(7):DOI 10.1186/s41043-0150009-x.

36. Newby P, Katherine L, Tucker K. Empirically derived eating patterns using factor or cluster analysis: a review. Nutr Rev. 2004;62(177-203).

37. Wang Y, Popkin B, Zhai F. The nutritional status and dietary pattern of Chinese adolescents, 1991 and 1993. Europian Journal of Clinical Nutrition. 1998;52(12):908-16.

38. Abraham D, Elifaged H, Berhanu E. Age-related factors influencing the occurrence of undernutrition in northeastern Ethiopia. BMC Public Health. 2015;15(108):17: DOI 10.1186/s12889-015-1490-2.

39. Tilahun A, Abraham D, Berhanu E. Prevalence and factors associated with undernutrition and anaemia among school children in Durbete Town, northwest Ethiopia. Archives of Public Health. 2015;73(34):1-7.

African Health Sciences Vol 18 Issue 3, September, 2018 
40. Jee HR, Aidan AC, Bhupendra B, Victor MA, Suzanne C, Sarah A. Household sanitation and personal hygiene practices are associated with child stunting in rural India: a cross-sectional analysis of surveys. BMJ Open. 2015;5: e005180. doi:10.1136/bmjopen-2014-005180.

41. Hunegnaw M, Takele T, Teresa K. Malnutrition and its Correlates among Rural Primary School Children of Fogera District, Northwest Ethiopia. Journal of Nutrition and Therapy. 2013;S12: 002. doi:10.4172/2161-0509.S12002.

42. Robert A, James OM, Thomas T. Determinants of Household Food Security in the Sekyere-Afram Plains District of Ghana.1st Annual International Interdisciplinary Conference, AIIC 2013, 24-26 April, Azores, Portugal -Proceedings-.eujournal.org/index.php/esj/article/ download/1488/1497.dawnload on 9/17/2015. 2013.

43. Ajao K, Ojofeitimi E, Adebayo A, Fatusi A, Afolabi O. Influence of Family Size, Household Food Security Status, and Child Care Practices on the Nutritional Status of Under-five Children in Ile-Ife, Nigeria. African Journal of Reproductive Health. 2010;14(4):1 23.
44. Andualem A, Abera K. Assessment of the impact of latrine utilization on diarrhoeal diseases in the rural community of Hulet Ejju Enessie Woreda, East Gojjam Zone, Amhara Region. Ethiop J Health Dev. 2010;24(2):110-8.

45. Checkley W, Gilman R, Black R. Effect of water and sanitation on childhood health in a poor Peruvian peri-urban community. Lancet. 2004;363:112-18.

46. Lin A, Arnold B, Afreen S. Household environmental conditions are associated with enteropathy and impaired growth in rural Bangladesh. Am J Trop Med Hyg. 2013;89(130-7).

47. Humphrey J. Child undernutrition, tropical enteropathy, toilets, and handwashing. Lancet. 2009;374(1032-5).

48. Ulrich ES, Stefan HEK. Malnutrition and Infection:Complex Mechanisms and Global Impacts. PLos Medicine. 2007;4(5): 0806-12. e115.

49. Vanessa SL, Israel CdLP, RisiaCristina EdM, OliveiraJuliana S, LeopoldinaAugusta dSS, SôniaLúciaLucena $\mathrm{SdA}$, et al. Factors associated with the decline in stunting among children and adolescents in Pernambuco, Northeastern Brazil. Rev Saúde Pública. 2012;46(2):2-8. 